JOURNAL OF SYNCHROTRON RADIATION

ISSN 1600-5775
Keywords: free-electron laser (FEL); PhotonDiag2017 workshop; photon diagnostics; beamline instrumentation; beamline design

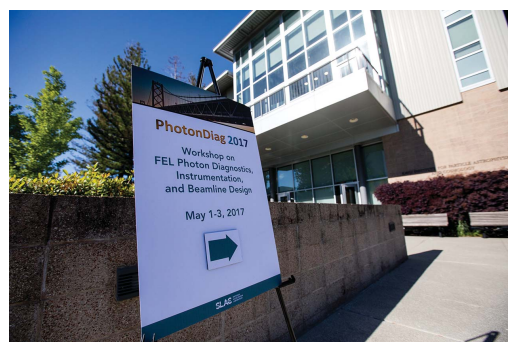

(C) 2018 International Union of Crystallography

\section{PhotonDiag2017 workshop: introductory overview}

\author{
Daniele Cocco, $^{\mathrm{a} *}$ Stefan Moeller, ${ }^{\mathrm{a} *}$ Elke Ploenjes $^{\mathrm{b} *}$ and Marco Zangrando ${ }^{\mathrm{c}, \mathrm{d}} *$
}

aSLAC National Accelerator Laboratory, 2575 Sand Hill Road, Menlo Park, CA 94025, USA,

${ }^{\mathbf{b}}$ Deutsches Elektronen-Synchrotron DESY, Notkestrasse 85, 22607 Hamburg, Germany,

'Elettra-Sincrotrone Trieste SCpA, SS 14 km, 163.5 in Area Science Park, Basovizza 34149, Italy, and

dIOM-CNR, SS 14 km, 163.5 in Area Science Park, Basovizza 34149, Italy. *Correspondence e-mail:

cocco@slac.stanford.edu,smoeller@slac.stanford.edu, elke.ploenjes@desy.de, marco.zangrando@elettra.eu

This issue of the Journal of Synchrotron Radiation is a special issue including papers from the PhotonDiag2017 workshop. Here, a brief introduction is given.

This special issue of Journal of Synchrotron Radiation includes a selection of presentations given at the Workshop on FEL Photon Diagnostics, Instrumentation and Beamline Design (PhotonDiag2017) hosted by the SLAC National Accelerator Laboratory, in Stanford, California, USA, on 1-3 May 2017, and co-sponsored by the FELs of Europe consortium.

Free-electron lasers (FELs) for the soft and hard X-ray range are fourth-generation light sources capable of producing high-brightness light pulses, ten billion times more intense than those emitted by synchrotrons, and of very short duration. These novel $\mathrm{X}$-ray sources with unprecedented beam properties have been enabling new scientific investigations in ultrafast science in the X-ray region. Besides the already existing FEL facilities such as FLASH (in operation since 2005), LCLS (2009), FERMI (2010) and SACLA (2011), recently a whole suite of FELs have completed their construction phase and initiated user operation: XFEL-Pohang (2016), SwissFEL (2016/17) and the Dalian Coherent Light Source (2016/2017), and most recently the European XFEL (2017). Meanwhile, upgrades of already existing FELs are underway, FLASH II (first lasing 2014) and LCLS II (2020).

But the FELs are not the only novel X-ray source development presently. Diffractionlimited storage rings (DLSRs) are under construction or in commissioning around the world. Through upgrade projects, like ALS-U or APS-U, or new constructions, like MAX IV or Sirius, the DLSRs will compete with FELs as X-ray sources for enabling more advanced science and experiments.

A central area of research and development, to fully exploit the potential of FELs and DLSRs, is focused on characterizing these exceptional photon sources in all their beam

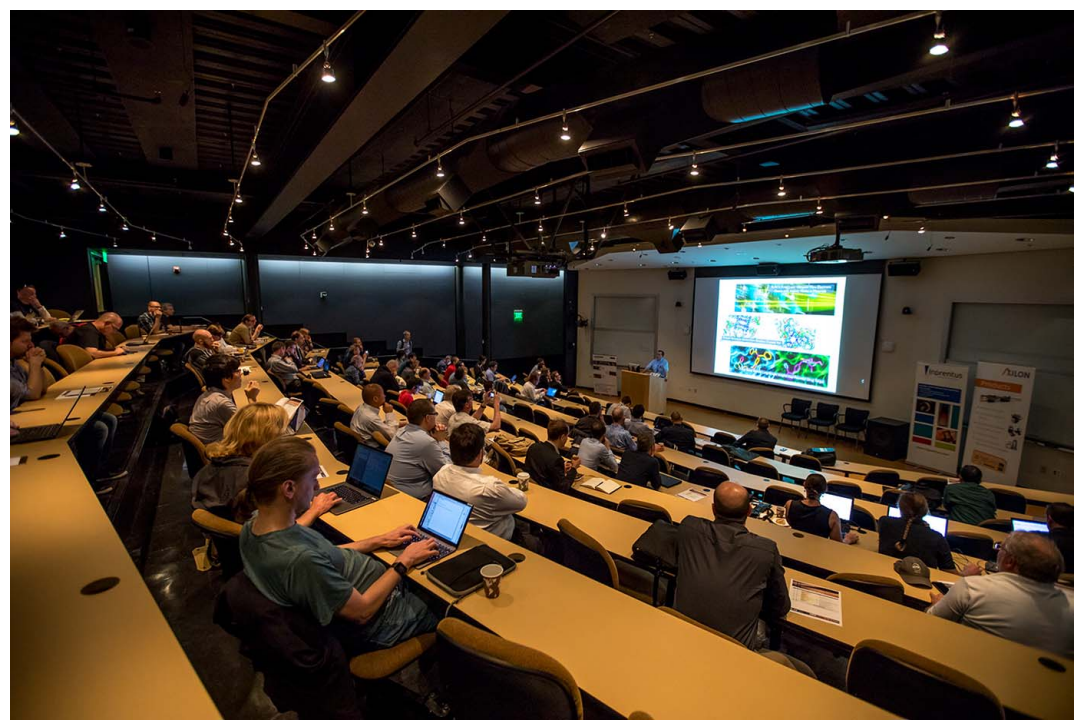

Photograph from the welcoming presentation given by the LCLS director Mike Dunne. 
properties, and transporting them to the experimental stations. This is the subject of this workshop series on FEL Photon Diagnostics, Instrumentation and Beamline Design, named 'PhotonDiag'. It is organized regularly as an activity of the Collaboration of European FEL and SPS Facilities (FELs of Europe). To extend the collaboration activities beyond Europe, for the first time, LCLS, the Linac Coherent Light Source at the SLAC National Accelerator Laboratory, organized and hosted, in collaboration with FELs of Europe, the third edition of the workshop, PhotonDiag2017. The three-day international event took place on 1-3 May 2017 on the SLAC campus with an attendance of over 100 scientists. PhotonDiag2017 is a follow-up of PhotonDiag2015 organized by Elettra-Sincrotrone Trieste (Trieste, Italy) and PhotonDiag2010 at DESY (Hamburg, Germany).

The workshop gave a comprehensive overview of the current state of recent results and ongoing developments in this exciting field of FELs and also DLSR science spanning topics from new facilities, novel accelerator machine modes to optical components, instrumentation and beam diagnostics. A special emphasis was placed on the challenges created by high-repetition-rate FEL operations, as well as pulse length measurements, synchronization with optical lasers and arrival time diagnostics among other topics.

We gratefully acknowledge the support of SLAC National Accelerator Laboratory, the FELs of Europe, and all our generous sponsors. We thank the LCLS staff for hosting and organizing this workshop. We thank the editorial staff of the Journal of Synchrotron Radiation for their efforts in publishing this special issue. We would also like to thank the Program Committee for their support in various aspects of the program's organization, and the session chairs for their help in running the conference. Without the participants and their high-quality scientific contributions, the success of this year's PhotonDiag2017 meeting would not have been possible. 